\title{
ESCAPE FROM FINITE INTERVALS: NUMERICAL STUDIES OF ORDER STATISTICS*
}

\author{
BARTŁOMIEJ DyBieC \\ The Marian Smoluchowski Institute of Physics \\ and \\ Mark Kac Center for Complex Systems Research, Jagiellonian University \\ Reymonta 4, 30-059 Kraków, Poland
}

(Received January 17, 2014)

The subdiffusive systems are characterized by the diverging mean residence time. The escape of a subdiffusive particle from finite intervals cannot be characterized by the mean exit time. The situation significantly changes when instead of a single subdiffusive particle there is an ensemble of subdiffusive particles. In such a case, if the ensemble of particles is large enough, the mean minimal first escape time (first exit time of the fastest particle) is well defined quantity and the minimal first exit time distribution has fast decaying power-law asymptotics. Consequently, the increase in the number of particles facilitates escape kinetics and shortenes the system's lifetime.

DOI:10.5506/APhysPolB.45.1037

PACS numbers: 05.40.Fb, 05.10.Gg, 02.50.-r, 02.50.Ey

\section{Introduction}

In the theory of stochastic systems, the escape of a Brownian particle is a crucial ingredient (element) of many phenomena. Usually, it is assumed that stochastic systems consist of a single particle coupled to the thermal bath and properties of diffusive motion of this particle are studied. Here, we continue the line of investigation initiated in [1], where the influence of coherent multi-particle dynamics on continuous time random walks $[2,3]$ was studied. In particular, in [1] it was shown that if an ensemble of particles moves coherently, the properties of such motion are sensitive to the actual number of particles. Consequently, we explore how the properties of the escape from finite interval can be affected by the number of diffusing particles [4-9].

* Presented at the XXVI Marian Smoluchowski Symposium on Statistical Physics, "Complexity of Brain - Critical Behaviour and Scaling", Kraków, Poland, August 28-31, 2013. 
Many experiments can be seen as the first exit/escape process [10]. Any detector records an event if a particle or signal reaches the detector. Consequently, the process of reaching the detector can be seen as the first exit/hitting problem. In reality, usually more than one particle is released. In principle, any of released particles can be detected but in many practical situations the detection of the first (fastest) particle is sufficient to record an event. The presence of many released particles changes the dynamics of the first detection significantly. In such realms, the first event (detection) is observed much faster than in the case when a single particle is released. More specifically, multi-particle dynamics can result in the finite mean minimal first exit time even in subdiffusive systems which due to trapping events cannot be characterized by the mean first exit time.

The next section presents the model of escape of a free particle from finite intervals both in the single particle and many particles regimes. Additionally, basic information about order statistics is included. In Sec. 3, the results of numerical simulations are compared with the theoretical predictions, also in the limit of infinite systems. The paper is closed with summary and conclusions (Sec. 4).

\section{Model and basic concepts}

Escape from finite intervals is an example of the process when the multiparticle dynamics of the same system can be significantly different from its single particle regime. In order to characterize the escape from finite intervals, first the single particle system is introduced. Next, the assumption that there is only one particle in the system is relaxed. Using the concept of the order statistics and the survival probability it is shown how presence of many particles can accelerate first escape process and hide anomalous diffusion.

\subsection{Single particle model}

A single subdiffusive $[3,11]$ particle is moving on the finite $[-L, L]$ interval restricted by two absorbing boundaries. In the continuous time random walk scenario [3, 11], the evolution of the probability density of finding a particle at time $t$ around $x$ is described by the time-fractional SmoluchowskiFokker-Planck equation [3, 11, 12]

$$
\frac{\partial p(x, t)}{\partial t}=\frac{\sigma^{2}}{2}{ }_{0} D_{t}^{1-\nu}\left[\frac{\partial^{2}}{\partial x^{2}} p(x, t)\right],
$$

with the initial condition $p(x, 0 \mid 0,0)=p(x, 0)=\delta(x)$, i.e. a particle starts its motion in the middle of the interval. Due to absorbing boundaries at $\pm L$, the probability density vanishes at the boundaries, i.e. $p(x= \pm L, t)=0$. In 
Eq. (1), ${ }_{0} D_{t}^{1-\nu}$ represents the Riemann-Liouville fractional time derivative defined by the relation

$$
{ }_{0} D_{t}^{1-\nu} f(x, t)=\frac{1}{\Gamma(\nu)} \frac{d}{d t} \int_{0}^{t} d t^{\prime} \frac{f\left(x, t^{\prime}\right)}{\left(t-t^{\prime}\right)^{1-\nu}} .
$$

In the limit of $\nu=1$, the fractional time derivative ${ }_{0} D_{t}^{1-\nu}$ vanishes and the time-fractional Smoluchowski-Fokker-Planck equation (1) reduces to the standard Smoluchowski-Fokker-Planck equation [13].

The solution of Eq. (1) can be constructed by separation of variables $[3,14]$ and it reads

$$
p(x, t)=\sum_{i=1}^{\infty} \frac{1}{L} \sin \left[\frac{i \pi}{2}\right] \sin \left[\frac{i \pi(x+L)}{2 L}\right] E_{\nu}\left[-\frac{i^{2} \pi^{2} \sigma^{2}}{4 L^{2}} t^{\nu}\right],
$$

where $E_{\nu}(\ldots)$ is the Mittag-Leffler function [15]

$$
E_{\nu}\left(-\lambda_{i} t^{\nu}\right) \equiv \sum_{j=0}^{\infty} \frac{\left(-\lambda_{i} t^{\nu}\right)^{j}}{\Gamma(1+\nu j)},
$$

with $\lambda_{i}=\frac{i^{2} \pi^{2} \sigma^{2}}{4 L^{2}}(i>0)$ being eigenvalues of the Laplace operator on a finite interval [14]. The Mittag-Leffler function generalizes the standard exponential function (for $\nu=1$ the Mittag-Leffler function reduces to the exponential function). The Mittag-Leffler function interpolates between stretched exponential (small $t)$

$$
E_{\nu}\left(-\lambda_{i} t^{\nu}\right) \propto \exp \left[-\frac{\lambda_{i} t^{\nu}}{\Gamma(1+\nu)}\right]
$$

and a power-law (large $t$ )

$$
E_{\nu}\left(-\lambda_{i} t^{\nu}\right) \propto \frac{t^{-\nu}}{\lambda_{i} \Gamma(1-\nu)}
$$

forms, see $[3,16]$.

As the main characteristics of the escape process the first exit time $\tau$ and its distribution $f(\tau)$ are used. From Eq. (1) the first passage time density $f(t)$ can be calculated [10, 13]

$$
f(t)=-\frac{d}{d t} \int_{-L}^{L} p(x, t) d x=-\frac{d}{d t} S(t),
$$

where $p(x, t)$ is the solution of Eq. (1). The survival probability 


$$
S(t)=\int_{-L}^{L} p(x, t) d x
$$

is the probability that at time $t$ a particle is still in the $[-L, L]$ interval. The survival probability $S(t)$ is the complementary cumulative distribution function

$$
S(t)=1-\mathcal{F}(t)=1-\int_{0}^{t} f(u) d u
$$

of the first passage time density. From Eq. (3), the survival probability can be calculated

$$
S(t)=\sum_{i=1}^{\infty} \frac{4}{i \pi} \sin ^{3}\left[\frac{i \pi}{2}\right] E_{\nu}\left[-\frac{i^{2} \pi^{2} \sigma^{2}}{4 L^{2}} t^{\nu}\right],
$$

and the first passage time density as $f(t)=-\frac{d}{d t} S(t)$. For $\nu<1$, the first passage time density has the power-law asymptotics $\left(f(t) \propto t^{-(\nu+1)}\right)$, while for $\nu=1$ the Mittag-Leffler function is replaced by the exponential function and the first passage time density has the exponential asymptotics determined by the smallest eigenvalue of the Laplace operator. In such a case, the mean first passage time $\langle\tau\rangle$ from a bounded (restricted) interval is finite and equals

$$
\langle\tau\rangle=\int_{0}^{\infty} S(t) d t=\int_{0}^{\infty} f(t) t d t=\frac{L^{2}}{2 \sigma^{2}} .
$$

For $\nu<1$, due to heavy tailed asymptotics of the first passage time density, the mean first passage time diverges. Nevertheless, the first passage time density (as well as the survival probability) is well and uniquely defined.

For a more general initial condition, i.e. $p(x, 0)=\delta\left(x-x_{0}\right)$, the survival probability attains the following form

$$
S\left(t \mid x_{0}\right)=\sum_{i=1}^{\infty} \frac{4}{i \pi} \sin \left[\frac{i \pi\left(x_{0}+L\right)}{2 L}\right] \sin ^{2}\left[\frac{i \pi}{2}\right] E_{\nu}\left[-\frac{i^{2} \pi^{2} \sigma^{2}}{4 L^{2}} t^{\nu}\right] .
$$

Finally, when initial conditions are uniformly distributed over $[-L, L]$ interval, the average survival probability is given by

$$
\langle S(t)\rangle=\frac{8}{\pi^{2}} \sum_{i=0}^{\infty} \frac{1}{(2 i+1)^{2}} E_{\nu}\left[-\frac{(2 i+1)^{2} \pi^{2} \sigma^{2}}{4 L^{2}} t^{\nu}\right] .
$$

In such a situation, due to random initial conditions escape is facilitated, but the asymptotics remains unaffected. 


\subsection{Multi-particle model and order statistics}

Single particle dynamics can be easily extended to the multi-particle dynamics. Now, instead of a single particle moving within the interval, there is an ensemble of $N$ independent particles which are released at the same point within a infinitesimally short time. The escape of $N$ particles from the finite interval is characterized by $N$ first passage times: $\left\{\tau_{1}, \tau_{2}, \ldots, \tau_{N}\right\}$. The quantity of central interest is the minimum of these $N$ first exit times

$$
\tau_{(1)}=\tau_{\min }=\min \left\{\tau_{1}, \tau_{2}, \ldots, \tau_{N}\right\},
$$

whose probability density can be easily calculated by use of the order statistics [17] or by the properties of the survival probability.

For $N$ independent, identically distributed random variables $Y_{1}, Y_{2}, \ldots, Y_{N}$ the first (smallest) order statistics is defined as

$$
Y_{(1)}=\min \left\{Y_{1}, Y_{2}, \ldots, Y_{N}\right\} \text {. }
$$

The cumulative density $\mathcal{F}_{N}^{\min }(y)$ of the first order statistics $Y_{(1)}$ can be found by the definition [17]

$$
\begin{aligned}
\mathcal{F}_{N}^{\min }(y) & =\operatorname{Prob}\left(Y_{(1)} \leqslant y\right) \\
& =1-\operatorname{Prob}\left(Y_{(1)}>y\right) \\
& =1-\operatorname{Prob}\left(Y_{1}>y, \ldots, Y_{N}>y\right) \\
& =1-\operatorname{Prob}\left(Y_{1}>y\right) \times \ldots \times \operatorname{Prob}\left(Y_{N}>y\right) \\
& =1-[1-\mathcal{F}(y)]^{N},
\end{aligned}
$$

where $\mathcal{F}(y)$ is the cumulative density function of $Y$. Equation (15) can be rewritten using the survival probabilities

$$
S_{N}^{\min }(t)=[S(t)]^{N},
$$

where $S(t)$ and $S_{N}^{\min }(t)=S_{N}^{(1)}(t)$ are survival probabilities for a single particle and $N$ particles systems respectively.

Equation (16) can be derived by use of the survival probability only. $S_{N}^{\min }(t)$ is the probability that all $N$ particles are still in the system $([-L, L]$ interval). Since motion of particles is assumed to be independent, the survival probability $S_{N}^{\min }(t)$ is the product of the single particle survival probabilities $S(t)$.

The minimal first passage time density $f_{N}^{\min }(t)$ is the time derivative of the minimal first passage time cumulative density $\mathcal{F}_{N}^{\min }(t)$. The mean minimal first passage time can be calculated as

$$
\left\langle\tau_{\min }\right\rangle_{N}=\left\langle\tau_{(1)}\right\rangle_{N}=\int_{0}^{\infty} S_{N}^{\min }(t) d t=\int_{0}^{\infty}[S(t)]^{N} d t .
$$


The survival probability $S(t)$ is a bounded $(1 \geqslant S(t)>0)$, decreasing function of time. This implies that $S_{N}^{\min }(t)<S(t)$ for all $t>0$. Consequently, except the situations when the mean minimal exit time diverges, $\left\langle\tau_{\min }\right\rangle_{N}$ decreases with the increasing number of particles $N$, see [4-9]. For every value of the subdiffusion parameter $\nu$, there is such a number of particles $N$ that the mean minimal exit time becomes finite. More precisely, for $N \nu>1$, the mean minimal first passage time becomes finite and for $N \nu>2$, the variance of the minimal first passage times is finite.

The $N$ first passage times can be sorted in the non-decreasing order

$$
\tau_{\min }=\tau_{(1)} \leqslant \tau_{(2)} \leqslant \ldots \tau_{(k)} \leqslant \ldots \tau_{(N)}=\tau_{\max } .
$$

$\tau_{(1)}$ is the time when one (the fastest) particle out of $N$ is absorbed at the boundary. At $\tau_{(k)}, k$ particles out of $N$ leave the system. Finally, $\tau_{(N)}$ is the time when last (the slowest) particle escapes from the system, i.e. it is the time when all particles become absorbed.

Analogously to the first order statistics, it is possible to calculate cumulative density of the maximum ( $N^{\text {th }}$ (largest) order statistic)

$$
\mathcal{F}_{N}^{\max }(y)=[\mathcal{F}(y)]^{N},
$$

and $k^{\text {th }}$ order statistics

$$
f_{N}^{(k)}(y)=N f(y)\left(\begin{array}{c}
N-1 \\
k-1
\end{array}\right)[\mathcal{F}(y)]^{k-1}[1-\mathcal{F}(y)]^{N-k},
$$

where $f(y)$ is the probability density function of $Y$, i.e. $f(y)=\frac{d \mathcal{F}(y)}{d y}$. Since, we are interested in the dynamics of the fastest particle and minimum has the "lightest asymptotics", it is used for further considerations only.

\section{Results}

The presented results were constructed by the method of subordination [18] which provides the stochastic representation of solutions of Eq. (1). The values of the Mittag-Leffler functions were calculated by the Matlab routine [19]. Moreover, by the appropriate time rescaling results for various interval half-width $L$ can be transformed into each other. More precisely, the $L$ half-width case can be transformed into the $L_{0}$ half-width case by the time rescaling $t \rightarrow t^{\prime}=t /\left(L / L_{0}\right)$, see Eq. (10). Therefore, without any loss of generality, in Figs. 1-3 results corresponding to $L=2$ are depicted. Additional information about parameters of simulations is included in figures' captions.

Figure 1 presents survival probabilities $S_{N}^{\min }(t)$ for the subdiffusion parameter $\nu=0.7$. Various curves correspond to various number of particles $N=\{1,2,3,4,5\}$. The solid line shows the theoretical single particle 
survival probability $S(t)$, while the dashed line demonstrates the long time asymptotics of the survival probability (exactly of the Mittag-Leffler function). With increasing number of particles $N$, the decay of the survival probability $S_{N}^{\min }(t)$ becomes faster, i.e. tails of the survival probabilities decay as $t^{-N \nu}$.

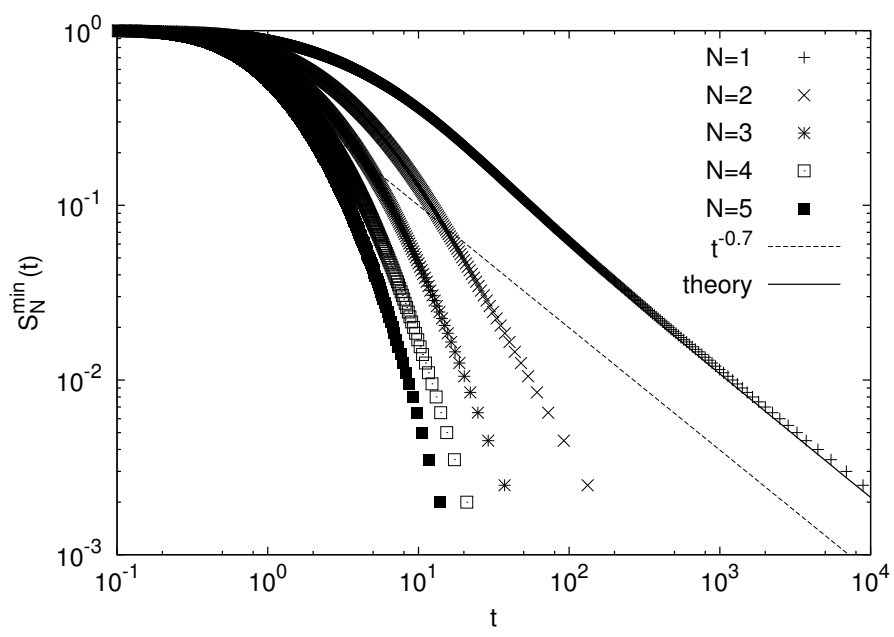

Fig. 1. Survival probabilities $S_{N}^{\min }(t)$ for the subdiffusion parameter $\nu=0.7$. Results are constructed by the subordination method with the time step of integration $\Delta t=10^{-3}$ and averaged over $10^{6}$ realizations. Various points correspond to various number of particles $N=\{1,2,3,4,5\}$. Dashed line shows $t^{-\nu}$ long time asymptotics of a single particle survival probability $S(t)$.

Figures 2 and 3 demonstrate rescaled survival probabilities. More precisely, instead of survival probabilities $S_{N}^{\min }(t)$ rescaled curves $\left[S_{N}^{\min }(t)\right]^{1 / N}$ are depicted. Such a rescaling superimpose all curves on top of each other making them indistinguishable, because for independent particles $S_{N}^{\min }(t)=$ $[S(t)]^{N}$. For every value of $\nu<1$, the asymptotics of the survival probability is of the power-law type, i.e. $S_{N}^{\min }(t) \propto t^{-N \nu}$. However, the transition point between stretched exponential and power-law regimes shifts towards longer times with increasing subdiffusion parameter $\nu$. Finally, for $\nu=1$ the system is Markovian and the survival probability has exponential asymptotics, see Fig. 3.

In Figs. 2 and 3 solid lines represent the exact $S(t)$ solution, see Eq. (10), which perfectly agrees with results of numerical simulations. The dashed lines in Fig. 2 show long time asymptotics of the survival probability which for $\nu<1$ is of the power-law type, i.e. $S_{N}^{\min }(t) \propto t^{-N \nu}$. 

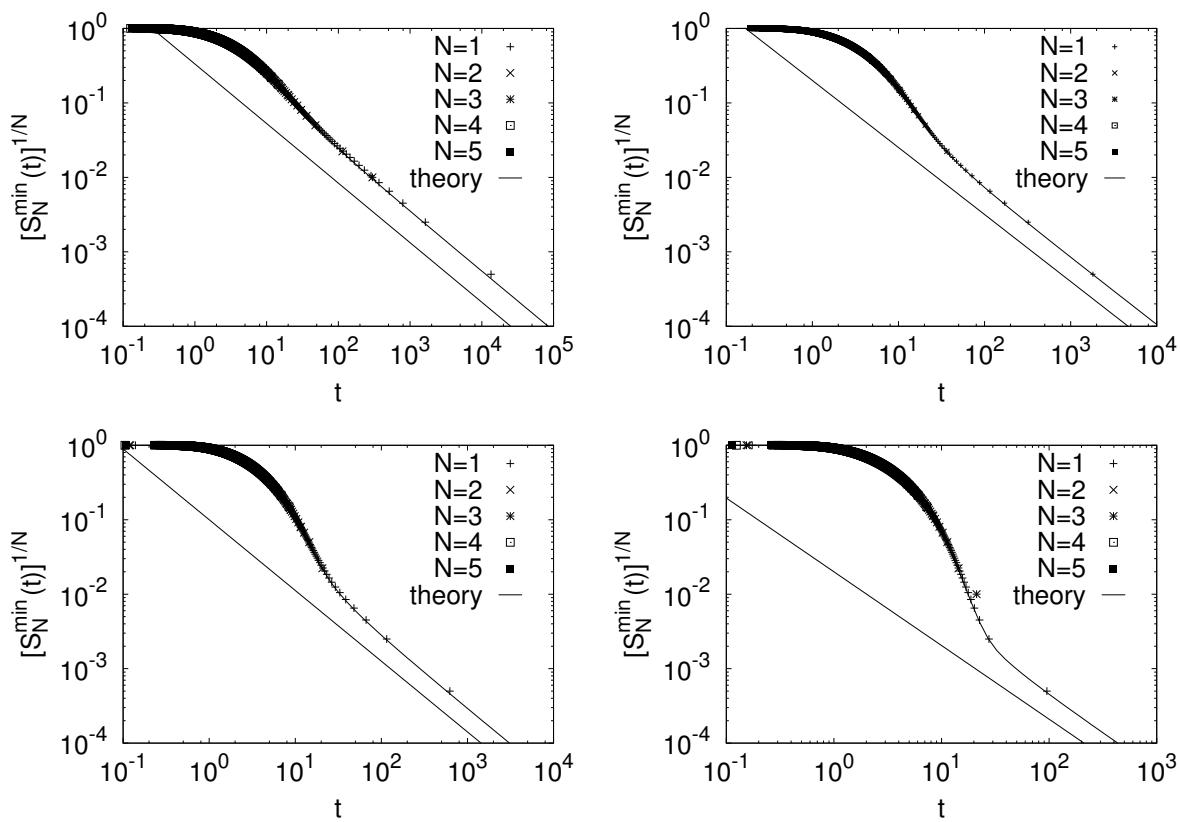

Fig. 2. Rescaled survival probabilities $\left[S_{N}^{\min }(t)\right]^{1 / N}$ for various values of subdiffusion parameter $\nu=0.8$ (top left panel), $\nu=0.9$ (top right panel) $\nu=0.95$ (bottom left panel) and $\nu=0.99$ (bottom right panel). Results are constructed by the subordination method with the time step of integration $\Delta t=10^{-3}$ and averaged over $10^{6}$ realizations.

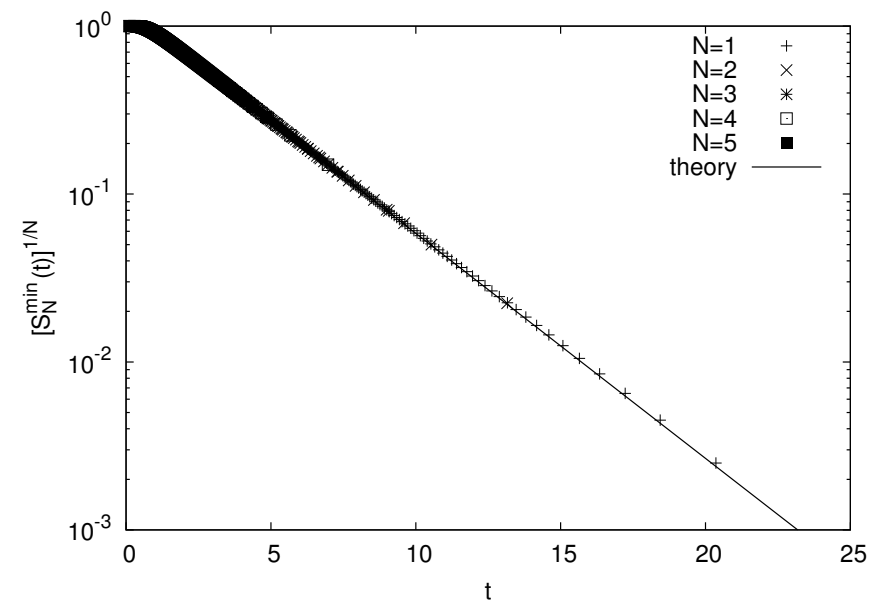

Fig. 3. Rescaled survival probabilities $\left[S_{N}^{\min }(t)\right]^{1 / N}$ for $\nu=1.0$. Results are constructed by the stochastic dynamics method with the time step of integration $\Delta t=10^{-4}$ and averaged over $10^{7}$ realizations. 
The interesting limiting behavior takes place when simultaneously $L \rightarrow \infty$ and $N \rightarrow \infty$ in such a way that the particle density is preserved, i.e. $\varrho=$ $N / 2 L=$ const. Assuming random initial conditions (uniformly sampled over over $[-L, L]$ interval) for $\nu=1$, one gets

$$
S_{\infty}^{\min }(t)=\lim _{N \rightarrow \infty L \rightarrow \infty}\langle S(t)\rangle^{N}=\exp \left[-\frac{2 \sigma \varrho}{\sqrt{\pi}} t^{1 / 2}\right],
$$

while in the more general case of $\nu<1$

$$
S_{\infty}^{\min }(t)=\exp \left[-\frac{\sigma \varrho}{\Gamma\left(1+\frac{\nu}{2}\right)} t^{\nu / 2}\right] .
$$

These results, up to the factor 2 in the exponent, are exactly the same as for an immobile target located at the origin surrounded by noninteracting traps performing random walks in continuous $1 \mathrm{D}$ space, see [7-9, 20]. The difference in the argument of exponent, $-\sigma \varrho t^{\nu / 2} / \Gamma\left(1+\frac{\nu}{2}\right)$ versus $-2 \sigma \varrho t^{\nu / 2} /$ $\Gamma\left(1+\frac{\nu}{2}\right)$, comes from the fact that escape of a particle from an $1 \mathrm{D}$ interval is equivalent to the motion of mobile traps on a circle with a single immobile target. Therefore, asymptotically two situations (i) escape from infinite interval and (ii) absorption at the origin, due to the same stretched exponential asymptotics, are hardly distinguishable.

The exact stretched exponential asymptotics of $S_{\infty}^{\min }(t)$ is difficult to access numerically, because one has to consider very large systems in order to keep $\langle S(t)\rangle \cong 1$. Otherwise, the limit survival probability is degenerate as it only takes values of 0 or 1 . Figure 4 shows numerically estimated survival probabilities for increasing systems' size with fixed particle density $\varrho=N / 2 L=0.1$ for $\nu=0.8$. The solid line represents exact asymptotics, i.e. $S_{\infty}^{\min }(t)=\exp \left[-a t^{\nu / 2}\right]$, which nicely agrees with numerical estimates.

Analogously, the stretched exponential asymptotics of the survival probability $S_{\infty}^{\min }(t)$ is reached in situations when jump lengths are generated from more general distributions [9], e.g. $\alpha$-stable density [21-24]. In such a case, evolution of the probability density is described by the bi-fractional Smoluchowski-Fokker-Planck equation [12, 18, 25]

$$
\frac{\partial p(x, t)}{\partial t}=\frac{\sigma^{\alpha}}{2}{ }_{0} D_{t}^{1-\nu}\left[\frac{\partial^{\alpha}}{\partial|x|^{\alpha}} p(x, t)\right]
$$

where $\frac{\partial^{\alpha}}{\partial|x|^{\alpha}}$ stands for the Riesz-Weil fractional derivative defined via the Fourier transform

$$
\mathcal{F}\left[\frac{\partial^{\alpha}}{\partial|x|^{\alpha}} f(x)\right]=-|k|^{\alpha} \hat{f}(x) .
$$




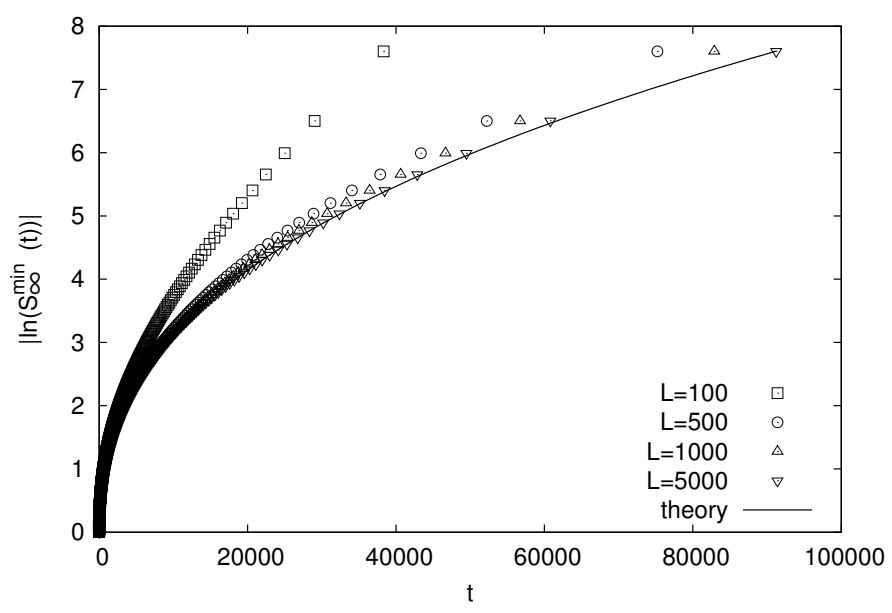

Fig. 4. Asymptotic survival probability $\left|\ln S_{\infty}^{\min }(t)\right|$ for $\nu=0.8$ and $\alpha=2.0$. Results are averaged over $10^{7}$ realizations. Solid line represents exact asymptotic survival probability, i.e. $\left|\ln \exp \left[-a t^{\nu / \alpha}\right]\right|$. Various points correspond to various system size. The particle density is fixed to $\varrho=N / 2 L=0.1$.

Exact asymptotics has the form $S_{\infty}^{\min }(t)=\exp \left[-a t^{\nu / \alpha}\right]$, see [9]. The $\nu / \alpha$ exponent is the manifestation of $\nu / \alpha$ self-similarity of the underlying random process [26]. This behavior is very well reproduced in numerical simulations. Figure 5 shows numerically estimated survival probabilities for increasing

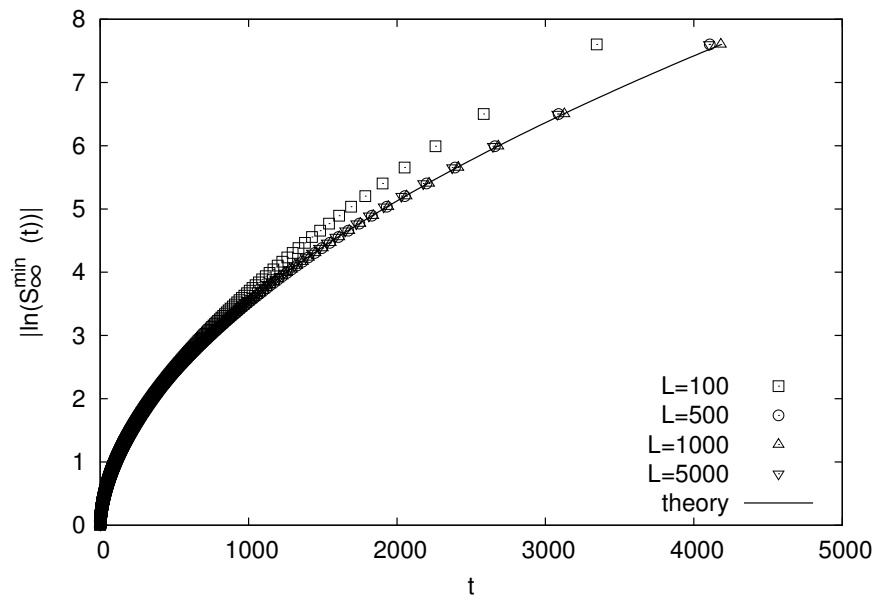

Fig. 5. Asymptotic survival probability $\ln \left|S_{\infty}^{\min }(t)\right|$ for $\nu=0.8$ and $\alpha=1.5$. Results are averaged over $10^{7}$ realizations. Solid line represents exact asymptotic survival probability, i.e. $\left|\ln \exp \left[-a t^{\nu / \alpha}\right]\right|$. Various points correspond to various system size. The particle density is fixed to $\varrho=N / 2 L=0.1$. 
systems' size with fixed particle density $\varrho=N / 2 L=0.1$. The solid line represents exact asymptotic results which perfectly agree with numerical estimates.

\section{Summary and conclusions}

A Brownian particle moving in a finite $[-L, L]$ interval escapes from the domain of motion with the probability 1 and the mean exit time is proportional to $L^{2}$. The situation drastically changes in the subdiffusive regime. Here, the particle still exits from interval with probability 1 , however the mean exit time becomes infinite. The divergence of the mean exit time is the direct consequence of the heavy tailed power-law asymptotics of the first exit time density. Nevertheless, there are some special conditions when detection of the escape of subdiffusive particles can be facilitated. Such a situation happens when two conditions are fulfilled simultaneously: (i) instead of a single particle the ensemble of $N$ subdiffusive particles is released and (ii) the escape event is recorded when at least one of particles (the fastest one) quits the domain of motion. In such a case, the minimal first passage time distribution for the finite number of particles still has the power law asymptotics with the exponent dependent on the number of particles released. More precisely, the exponent characterizing the decay of the minimal first passage time distribution increases linearly with the number of particles. Consequently, the minimal first passage time density despite being of the power-law type is characterized by the finite mean and the finite variance. In comparison to the mean, in order to make the variance of the minimal first passage time finite the larger number of particles is necessary.

Escape of an ensemble of $N$ independent particles from the finite interval is characterized by $N$ exit times $\left\{\tau_{1}, \ldots, \tau_{N}\right\}$. Among these exit times, there is the smallest $\left(\tau_{\min }=\tau_{(1)}\right)$ and the largest one $\left(\tau_{\max }=\tau_{(N)}\right) \cdot \tau_{(1)}$ informs when one can expect escape of a first (fastest) particle while $\tau_{(N)}$ provides information when all particles escape from the system (reach the absorbing boundary). $\tau_{(k)}$ characterizes intermediate regime, i.e. it estimates when $k$ out of $N$ particles can reach the boundary.

Probability density function of maximal escape time has the same asymptotics like the exit time distribution. Contrary to the maximal exit time, the minimal exit time has a distribution with lighter tails than the first passage time density. Therefore, with the increasing number of particles the mean minimal first passage time $\left\langle\tau_{\min }\right\rangle_{N}$ can become finite. The average maximal exit time $\left\langle\tau_{\max }\right\rangle_{N}$ is finite when the first exit time $\langle\tau\rangle$ is finite, otherwise it diverges. Escape of $k$ out of $N$ particles is in the between regime of escape of first (fastest) and last (slowest) particle. Consequently, it can be finite or diverge depending on the number of particles $k$, the total number of particles $N$ and the first passage time distribution. For scenarios de- 
scribed by Eq. (1) or (23) with $\nu<1$, the single particle survival probability has the $t^{-\nu}$ asymptotics. Therefore, $S_{N}^{\min }(t) \propto t^{-N \nu}, S_{N}^{\max }(t) \propto t^{-\nu}$, while $S_{N}^{(k)} \propto t^{-\nu(N-k+1)}$. The mean exit time of $k$ out of $N$ particles is finite when $\nu(N-k+1)>1$, the variance of first exit time distributions is finite when $\nu(N-k+1)>2$.

The very different situation takes place for infinite systems. For example, if the system size and the number of particles are simultaneously increased in such a way that the particle density is fixed, the survival probability attains exact asymptotic form which is of the stretched exponential type. In this limit, the survival probability is the same as the survival probability of immobile target (located at the origin) surrounded by mobile traps. The only one difference is in the prefactor in the argument of exponential function, because escape from interval restricted by two targets is equivalent to the motion of mobile traps on a circle with one target.

The mean (minimal) first passage time itself cannot be used to discriminate between normal and anomalous diffusion when more than one particle is released. Instead of the mean (minimal) exit time, the statistics of exit times should be studied. Nevertheless, it is necessary to be very careful because also the minimal first passage time density can be fast decaying function of time. Therefore, it can be hard to see its power-law asymptotics especially when a large number of particles is released.

Computer simulations have been performed at the Academic Computer Center Cyfronet, AGH University of Science and Technology (Kraków, Poland).

\section{REFERENCES}

[1] B. Dybiec, J. Phys. A: Math. Gen. 43, 312001 (2010).

[2] E.W. Montroll, G.H. Weiss, J. Math. Phys. 6, 167 (1965).

[3] R. Metzler, J. Klafter, Phys. Rep. 339, 1 (2000).

[4] S.B. Yuste, K. Lindenberg, J. Stat. Phys. 85, 501 (1996).

[5] J. Dräger, J. Klafter, Phys. Rev. E60, 6503 (1999).

[6] S.B. Yuste, L. Acedo, K. Lindenberg, Phys. Rev. E64, 052102 (2001).

[7] S.B. Yuste, K. Lindenberg, Phys. Rev. E72, 061103 (2005).

[8] S.B. Yuste, K. Lindenberg, Phys. Rev. E76, 051114 (2007).

[9] J. Franke, S.N. Majumdar, J. Stat. Mech. 2012, P05024 (2012).

[10] S. Redner, A Guide to First Passage Time Processes, Cambridge University Press, Cambridge 2001.

[11] R. Metzler, J. Klafter, J. Phys. A: Math. Gen. 37, R161 (2004). 
[12] R. Metzler, E. Barkai, J. Klafter, Europhys. Lett. 46, 431 (1999).

[13] C.W. Gardiner, Handbook of Stochastic Methods for Physics, Chemistry and Natural Sciences, Springer Verlag, Berlin 2009.

[14] D.R. Cox, H.D. Miller, The Theory of Stochastic Processes, Chapman and Hall, London 1965.

[15] I. Podlubny, Fractional Differential Equations, Academic Press, San Diego 1999.

[16] W.G. Glöckle, T.F. Nonnenmacher, Rheol. Acta 33, 337 (1994).

[17] H.A. David, H.N. Nagaraja, Order Statistics, John Wiley, New York 2003.

[18] M. Magdziarz, A. Weron, K. Weron, Phys. Rev. E75, 016708 (2007).

[19] I. Podlubny, M. Kacenak, Mittag-Leffler Function - Calculates the MittagLeffler Function with Desired Accuracy, MATLAB Central File Exchange, http://www . mathworks.com/matlabcentral/fileexchange/8738, 2009.

[20] R. Blythe, A. Bray, Phys. Rev. E67, 041101 (2003).

[21] W. Feller, An Introduction to Probability Theory and Its Applications, John Wiley, New York 1968.

[22] J.P. Nolan, Stable Distributions - Models for Heavy Tailed Data, Birkhäuser, Boston 2013, in progress, Chapter 1 online at http://academic2.american.edu/ jpnolan

[23] V.V. Yanovsky, A.V. Chechkin, D. Schertzer, A.V. Tur, Physica A 282, 13 (2000).

[24] D. Schertzer et al., J. Math. Phys. 42, 200 (2001).

[25] M. Magdziarz, A. Weron, Phys. Rev. E75, 056702 (2007).

[26] B. Dybiec, E. Gudowska-Nowak, Phys. Rev. E80, 061122 (2009). 